\title{
Un Primer Acercamiento al Docente frente a una Metodología Basada en Proyectos
}

\author{
Alma A. Benítez ${ }^{(1)}$ y Martha L. García ${ }^{(2)}$ \\ (1) Instituto Politécnico Nacional, CECyT 11. Wilfrido Massieu, Av. de los Maestros 217, Col. Casco de \\ Santo Tomás, CP 11340, México D. F.- México (e-mail: albenper@gmail.com). \\ (2) Instituto Politécnico Nacional, ESIME-ZACATENCO, Avenida Instituto Politécnico Nacional S/N, \\ Col. Zacatenco, Del. Gustavo A. Madero. D.F.-México (e-mail: martha.garcia@gmail.com).
}

Recibido Jul. 04, 2012; Aceptado Ago. 07, 2012; Versión final recibida Sep. 23, 2012

\begin{abstract}
Resumen
Considerando la evidente pérdida de interés por parte de los estudiantes por tópicos relacionados con la ciencia, el Instituto Politécnico Nacional ha promovido un modelo educativo centrado en el proceso aprendizaje del estudiante, cuya metodología es el Proyecto de Aula. La metodología ha sido implementada en el Centro de Estudios Científicos y Tecnológicos, siendo uno de los objetivos principales la creación de una cultura científica. Este estudio se centra en el desempeño de los docentes frente a ésta metodología y se identifican las habilidades que el profesor desarrolla cuando ha tenido ésta vivencia de proyecto en aula. Los resultados muestran que hay un desarrollo de habilidades cognitivas, cognitivalingüísticas, comunicativas y técnicas, aunque se presentaron situaciones donde se manifestaba la resistencia al cambio
\end{abstract}

Palabras clave: ciencia y tecnología, proyecto aula, habilidades, destrezas.

\section{A First Approach Teacher in front of a Methodology Based on Projects}

\begin{abstract}
Considering the evident loss of interest among students for scientific subjects, the National Polytechnic Institute in Mexico has promoted an educational model focused on the student learning process, using the methodology known as Classroom Project. The methodology has been implemented at the Center for Scientific and Technological Studies, being the development of scientific culture one of the main objectives. This study is centered on the performance of the instructors when using this methodology and on identifying the abilities that the professor learns when facing this method or classroom project. The results show that there is a development of cognitive, cognitive-linguistic, communicative and technical skills, although there were cases where resistance to change were still present.
\end{abstract}

Keywords: science and technology, classroom project, abilities, skills. 


\section{INTRODUCCIÓN}

Muchos nos hemos preguntado sobre; ¿Por qué el estudio de la ciencia no resulta atractivo para muchos alumnos? Buena parte de la responsabilidad recae en el planteamiento que mantiene su enseñanza, que sigue mostrando la mayor parte de las veces: i) Una imagen de ciencia centrada en sí misma, académica y formalista (Astudiño C., Rivarosa A. y Ortiz F., 2011); y ii) Falta de conexión de lo que se enseña con la ciencia que está presente en el mundo cotidiano o con la ciencia no formal de los medios de comunicación (Pro Bueno A. y Ezquerra A., 2008). En muchas escuelas del nivel bachillerato introducen al estudiante de ciencias el primer tema, por ejemplo, en física sobre una red de términos de alto nivel formal (desplazamiento, velocidad media, velocidad instantánea, aceleración instantánea, etc.), y que paradójicamente algunas de estas nociones, una vez propuestas, son ignoradas sin la más mínima aplicación posterior, dejando al estudiante confundido y preguntándose ¿cuál es la utilidad de tantas fórmulas en la práctica?.

Es evidente que los rasgos anteriormente citados no contribuyen a hacer la ciencia atractiva entre los jóvenes estudiantes. El resultado es una pérdida de interés hacia algo que se ve difícil, arbitrario y poco relacionado con la realidad, percepción particularmente grave si consideramos el papel primordial que juegan ciencia y tecnología en la sociedad de nuestros días. Ante estos hechos se insiste en la necesidad de una cultura científica como objetivo educativo fundamental y de la cual emanan las siguientes preguntas; ¿Cómo emprender un proyecto que incluya a los docentes y principalmente a los estudiantes?, ¿qué papel jugará el profesorado?, ¿cómo llevar a la práctica estas ideas?, diversos países (España, Reino Unido, Estados Unidos, entre otros) han abordado ésta tópicos promoviendo estrategias de enseñanza de las ciencias.

Por lo que es prioritario impulsar el conocimiento de la naturaleza de la ciencia como un contenido fundamental en planteamientos curriculares de la enseñanza de las ciencias. El Conocimiento Didáctico del Contenido desde la perspectiva Schwartz y Lederman, (2002), mencionado en el artículo de Acevedo D. (2009) presenta una propuesta teórica que permite vincular tres esferas fundamentales en la formación del docente de ciencia respecto al desarrollo de la práctica docente del Conocimiento de la Naturaleza, no obstante será la colaboración entre docentes en ejercicio e investigadores e investigadoras universitarios la que podrá generar las mejores indagaciones, ricas en consecuencias teóricas y prácticas, y las mejores abstracciones, que ofrezcan vías para mejor percibir, comprender y transformar la práctica (Korthagen, 2007). En este sentido, es necesario que el docente diseñe estrategias de aprendizaje encaminadas a la indagación científica, lo que implica un enfoque reflexivo para abordar diversos contextos de la ciencia mediante una enseñanza basada en proyectos que permita la inserción del estudiante en una sociedad que exige la solución de diversos problemas y el planteamiento de nuevas problemas (Aravena M., Caamaño C. y Giménez J., 2008).

El Instituto Politécnico Nacional (IPN) ha implementando un Modelo Educativo centrado en el estudiante, en donde se atiende de manera directa las necesidades que enfrente la sociedad en general, promoviendo una formación integral y de alta calidad científica, tecnológica y humanística, y que combine equilibradamente el desarrollo de conocimientos, actitudes, habilidades y valores (Materiales para la Reforma, IPN, 2004). EI Modelo Académico, derivado del Modelo Educativo, incluye dos aspectos básicos: a) la estructura organizacional y, b) los planes de estudio. En particular, en el Nivel Medio Superior los planes de estudio deben orientar la construcción de conocimiento a través de la resolución de problemas, la investigación, el trabajo en equipo y la búsqueda de información. Teniendo como propósito atender problemas en el ámbito social, en particular aquellos que impactan la Sustentabilidad del Medio Ambiente, en temas relacionados con la biodiversidad del planeta. Estos grandes cambios que se han producido en la sociedad deben ser atendidos de manera inmediata por la misma sociedad, siendo el recurso fundamental para lograrlo la Educación para un desarrollo sustentable, en especial para el medio ambiente (Lacueva A., 2010).

El proyecto de aula es una propuesta metodológica que permite incorporar los objetivos de las unidades de aprendizaje en un semestre a la solución de un problema, definido en un proyecto, lo que beneficia el desarrollo de diversas habilidades del pensamiento en el estudiante, abriendo nuevas oportunidades para impulsar enfoques innovadores de aprendizaje y de enseñanza, así mismo la nueva visión implica que el docente desarrolle nuevas habilidades y destrezas, ya que se verá sacudido por la misma problemática que el estudiante enfrentará, corregida y aumentada, principalmente porque habrá de asumir una metodología a la que, en general, no está acostumbrado, por este motivo, la metodología del Proyecto de Aula supone un esfuerzo considerable de adaptación. El presente trabajo expone la experiencia de un grupo de docentes que ha tenido la vivencia con esta metodología, en la cual se expone las habilidades generadas durante el proceso. 
Camarena (2009) señala, que el docente debe tener conocimientos sobre elementos psicológico, emocionales, cognitivos y psicológicos relacionados con sus estudiantes como intereses, valores, estilos de aprendizaje, maneras de comunicarse formas de relacionarse y de aprender, lo que también enfatiza Echeverría (2002), al señalar los elementos cognitivos, cognitivos lingüísticos, comunicativos y técnicos que el docente requiere para tener un mejor desempeño académico. Entre los elementos que señala Echeverría (2002) se encuentran: saber explicar, clasificar identificar, justificar, transferir, argumentar con claridad la hipótesis y conclusiones, resumir problemas esenciales de los problemas, formular la situación, tomar decisiones e interpretar los resultados en los contextos de origen del problema. Para concretar lo señalado por Camarena y Echeverría, de acuerdo con la metodología del Proyecto de Aula del IPN, se identifican en los profesores no solo cambio de estrategias de enseñanza que dan énfasis a contenidos conceptuales, sino habilidades: comunicativas, cognitivas lingüísticas, técnicas y valores.

En lo concerniente al fundamento de la metodología del Proyecto de Aula, es importante mencionar que ésta metodología tiene sus raíces en el enfoque constructivista, en cuyo escenario el aprendizaje implica; un proceso constructivo interno a partir de sus conocimientos previos, reconstrucción de saberes culturales, para lo cual requiere descontextualizaciones con tareas significativamente culturales, no obstante la enseñanza también experimenta una nueva visión, el docente es ahora un mediador entre el conocimiento y el aprendizaje, es un profesional reflexivo y promueve aprendizajes significativos (Enríquez M., Enríquez S. Pizano S., 2007). El proyecto de aula es una fuente que inspira la búsqueda y construcción de conocimiento, así como es el motor que impulsa al encuentro de soluciones de problemáticas del interés propio del estudiante. Es en este sentido que con la mediación de procesos de investigación, el desarrollo y logro del proyecto de aula propugnan por una actitud científica, bien sea visto a partir de la disciplina o profesión que se aborde. Las dinámicas de este tipo de proyectos exigen que los estudiantes trasciendan de la formulación de un problema y de la simple consulta bibliográfica y se vean en la necesidad de desarrollar habilidades para enfrentarse a los procesos, vivencias. Para incorporar al docente en ésta dinámica es necesario que domine su asignatura y conozca las áreas disciplinarias del contexto de los eventos a trabajar en el ambiente de aprendizaje. Ante este nuevo reto, el papel del docente se concibe dentro de un proceso dinámico en construcción permanente en donde participan todos los agentes educativos, que se requieran consolidar los espacios de reflexión en los que define la labor del docente.

\section{DISEÑO DEL ESTUDIO}

Esta investigación, se ubica en un paradigma de investigación cualitativo, cuya observación se llevó a cabo durante un semestre escolar para identificar las habilidades que el docente desarrolla cuando ha tenido ésta vivencia. Las observaciones en el estudio se desarrollaron en dos niveles: global y específico. El primer nivel se orientó a registrar los siguientes eventos:

a) Examen diagnóstico. Se aplicó a 9 docentes participantes un examen diagnóstico para identificar su perfil laboral, así como su experiencia docente en el centro educativo (CECYT 11). Es importante señalar que los docentes participaron en cursos-talleres impartidos por la Escuela para introducir y fortalecer la propuesta metodológica del Proyecto de Aula.

b) Bitácora de la experiencia. En cada una de las reuniones que acordaron los docente durante el desarrollo de las etapas que constituyen el Proyecto de Aula, el investigador anotó los hechos más relevantes durante las sesiones, posteriormente la información era analizada para la siguiente reunión, en particular, se tenía especial atención a las actividades que presentaron dificultad durante su desarrollo, las cuales se utilizaban como base para la discusión con el grupo de docentes.

c) Grabaciones de las sesiones. Específicamente cuando el docente exponían sus puntos de vista sobre los avances o bien las propuestas al equipo de docentes, para continuar con el desarrollo del proyecto

A nivel específico la observación se dirigió a examinar con mayor detalle los procesos que lleva a cabo durante el proceso del planteamiento del problema y del diseño de la investigación para llevarlo al aula desde su unidad de aprendizaje.

La triangulación de la información se llevó a cabo desde distintas perspectivas para fortalecer la credibilidad en los resultados e interpretación del estudio. Lo anterior se llevó a cabo a través de identificar los hallazgos que se encontraron en la fuente A (reporte escrito individuales del docentes), fuente B (discusión grupal entre los 9 docentes), fuente $C$ (reportes escrito sobre el avance del proyecto en el aula, de los docentes), y también puedo corroborarse con la fuente $D$ (observaciones en clase), permitiendo comparar información proveniente de diferentes escenarios. 


\section{EXPERIENCIA EDUCATIVA}

\section{Fase diagnóstica}

Sujetos de estudio. 9 docentes participaron en el proyecto titulado "Casa Ecológica Autosuficiente, tratamiento de aguas jabonosas y pluviales", que se impartió en el cuarto semestre del CECyT 11 y cuyas Unidades de Aprendizajes fueron: Cálculo Diferencial, Física General II, Química General II, Sistema y Proyectos Constructivos de Instalaciones, Dibujo Técnico II, Dibujo Arquitectónico asistido por Computadora, Biología, Altimetría, Entorno Socioeconómico de México y Gestión y Formación de Empresas Constructoras.

Se aplicó un cuestionario diagnóstico, cuyo propósito fue diagnosticar sobre los siguientes aspectos: a) Identificación de problemas de aprendizaje desde el puntos de vista; curricular, institucional, organizacional y en el aula, b) Estrategias de enseñanza que emplea el docente en el aula y c) los aprendizajes que se pueden promover con el uso de estrategias de enseñanza. Se diseño un cuestionario estructurado en tres secciones:

i) Datos personales del docente, años de servicio, grado de escolaridad, horas frente a grupo, unidades de aprendizaje impartidas y participación en cursos, seminarios, foros, congresos.

ii) Identificación de problemas en el aula. Aquí se les solicita expongan los problemas más significativos que consideren se presentan en el aprendizaje.

iii) Identificar estrategias; Para esta sección se solicita de acuerdo a su experiencia escriba estrategias que desde su experiencia han permitido superar las situaciones de aprendizaje que presenta el alumno.

Los docentes participantes tenían experiencia en el Nivel Medio Superior entre 5 y 25 años de servicio, su último grado de escolaridad es licenciatura y/o maestría, las horas frente a grupo fluctúan entre 15 a 25 horas por semana. Solo el $5 \%$ de los profesores habían participado en cursos y/o seminarios referentes a Investigación Educativa.

El examen diagnóstico arrojo diversas posiciones respecto a su quehacer educativo, señalando básicamente; la deficiencia de conocimientos, falta de interés (motivación), la necesidad de aprobar un curso (calificación) sin importar el conocimiento adquirido, lo que conlleva al desinterés del estudiante por las asignaturas, originando la indisciplina y el surgimiento de diversas estrategias para acreditarlas utilizando herramientas que son accesibles para el estudiante, es decir, trabajos o bien exposiciones de diversos temas ajenos a un interés genuino para culminar en transcripción y lectura de contenido no significativos.

Los profesores de acuerdo a su experiencia mencionan las estrategias de enseñanza que a su consideración ayuda a resolver las situaciones antes planteadas, por ejemplo; una de las estrategias más socorridas es llevar a cabo diversas pláticas con los estudiantes para comentarles sugerencias y que reflexionen sobre su situación. La estrategia permite establecer comunicación con los estudiantes, pero no se remedia el poco interés que el estudiante expone ante situaciones rutinarias en el aula, no obstante, dos docentes de 22 y 29 años de servicios, respectivamente, mencionan estrategias que son relevantes, como son; vincular los contenidos de las asignaturas con las vivencias cotidianas del estudiante considerando su contexto, discusiones grupales y el diseño de estrategias atendiendo los estilos de aprendizaje de los alumnos. Estrategias que son fundamentales en la Metodología de Proyecto de Aula. De acuerdo con lo expuesto, los docentes tienen conocimiento de las habilidades que requieren para su labor educativa, los cuales son mencionados por Echeverría (2002): Cognitivos (interpretar, analizar), Cognitivo-lingüístico (argumentar), Comunicativos: (hablar en público, defender sus puntos de vista), Técnicos (pronosticar).

\section{EXPERIENCIA: PROYECTO DE AULA}

El Proyecto de Aula se implementó a un grupo de 43 estudiantes de cuarto semestre de bachillerato (C.E.C.YT. 11) y cuya duración fue de 18 semanas, las edades de los estudiantes fluctuaban entre 16-17 años, el grupo participó en el proyecto titulado "Casa Ecológica Autosuficiente, tratamiento de aguas jabonosas y pluviales", cuyo objetivo fue: Diseñar y Construir una casa habitación aplicando la normatividad vigente para dar servicio autosuficiente de manera sustentable, en el cual participaron los 9 docentes antes mencionados. 


\section{El proyecto consta de 6 etapas:}

$1^{\circ}$ Etapa. Reunión de los 9 docentes, para dar inicio a la planeación del proyecto, partiendo de la discusión de los objetivos de aprendizaje y los contenidos de las unidades de aprendizaje involucradas. Durante las sesiones se consideró el eje temático: Medio Ambiente. Cada uno de los 9 docentes participantes elaboró una matriz de las competencias disciplinares, considerando que para el nivel de cuarto semestre se enfocó exclusivamente a una investigación de tipo tecnológico.

$2^{\circ}$ Etapa. Reunión de profesores, tutores y estudiantes para definir el tema del proyecto, la hipótesis, el aporte de cada asignatura, los productos esperados y las formas de evaluar el proyecto. En ésta etapa se delimitó la propuesta de investigación al Reciclaje, siendo el tema central la Casa Ecológica, en cuya investigación se dirigió al campo tecnológico, atendiendo la investigación documental y tecnológica para adquirir competencias disciplinares o genéricas según sea el caso para cada una de las unidades de aprendizaje.

$3^{\circ}$ Etapa. Cada docente elaboró su plan del proyecto, cubriendo los objetivos de aprendizaje.

$4^{\circ}$ Etapa. Reuniones para enlazar las actividades entre las asignaturas, calendarizar las sesiones para el control y evaluación participativa.

$5^{\circ}$ Etapa. Desarrollo de las actividades planeadas en cada asignatura, revisión del cumplimiento de los aprendizajes esperados.

$6^{\circ}$ Etapa. Se presenta la investigación y los resultados obtenidos a los coordinadores Generales, Académico y Administrativos, en plenarias para dar a conocer las experiencias obtenidas tanto en las fortalezas como en los obstáculos que se presentaron durante la experiencia. Dicha etapa es la más importante porque expresar los procesos en forma oral adquiriendo relevancia en las disciplinas, particularmente en un proyecto que ofrece numerosas ventajas para la enseñanza de los aprendizajes; por eso, se destaca que facilita el aprendizaje de matices en lo que se desea transmitir y que es difícil percibirlo de otra manera. Asimismo, hay descubrimientos que se producen al comunicar oralmente lo que los estudiantes saben: explicación de un proceso, justificaciones de cálculos, síntesis de sus conocimientos o propuesta de procedimientos (Aravena M., Caamaño C. y Giménez J., 2008).

\section{ANÁLISIS DE DATOS}

Las dimensiones que se aplicaron para llevar a cabo el análisis corresponden a las habilidades que el docente desarrollo durante la experiencia, son de diferente naturaleza:

i) Comunicativas en general (COM); relacionadas con el lenguaje oral.

ii) Cognitivos lingüísticas $(\mathrm{CL})$; relacionadas con cada una de las tipologías textuales que se emplean para comunicar la ciencia.

iii) Cognitivas (C); habilidades del pensamiento.

iv) Técnicas (T): relacionadas con el manejo de los contenidos del tema que se está abordando.

De lo anterior se desprende la importancia de la metodología a seguir para poder desarrollar las habilidades requeridas frente a los retos que presenta actualmente la educación. De acuerdo con los resultados obtenidos en el examen diagnóstico, reportes individuales y grupales, además de las discusiones con el grupo de docentes, se puede plantear que los docentes participantes muestran el fortalecimiento de algunos de los elementos que requieren para la realización de su tarea como docente en el proyecto de aula (Echeverría, 2002 y Camarena, 2009). La tabla 1 expone el análisis que arrojaron las observaciones, las entrevistas y cuestionarios, realizados durante la experiencia educativa.

\section{DISCUSIÓN}

Los docentes muestran un reconocimiento global para el diseño del proyecto, y la participación de la unidad de aprendizaje, reconocen los tiempos limitados del semestre y ajustan tiempos para cubrir el programa, sin descuidar el proyecto. Se destaca la organización en la planificación y diseño del proyecto, ya que el grupo plantea diversas presentaciones mostrando la información recopilada y muestran los diversos estudios relacionados con la unidad de aprendizaje de medición e instrumentación electrónica. Durante la experiencia el equipo de docentes presentó diferentes puntos de vista sobre el proyecto, para establecer consensos que permitió el avance del proyecto, desarrollando un ambiente de discusión y de acuerdos constantes. 
De lo anterior se desprende la necesidad de que el docente se capacite en una serie de temas fundamentales para el buen desempeño académico, que repercute en el enriquecimiento de las unidades de aprendizaje y en el desarrollo de las habilidades de los estudiantes.

Tabla 1. Habilidades Identificadas

\begin{tabular}{|c|c|c|}
\hline \multicolumn{2}{|c|}{ Habilidades } & Descripción \\
\hline \multirow[t]{4}{*}{ Etapa 1} & C & $\begin{array}{l}\text { Idea vaga del tema a participar del Eje Temático establecido. } \\
\text { Reconoce la necesidad de participar con su unidad de aprendizaje en el eje temático: Medio } \\
\text { Ambiente. } \\
\text { Idea vaga de la participación de las unidades de aprendizaje en el proyecto. } \\
\text { Analiza y discute la organización de su unidad de aprendizaje para participar en el proyecto. }\end{array}$ \\
\hline & $\mathrm{CL}$ & $\begin{array}{l}\text { Discusión en mesa de trabajo para analizar los diferentes contenidos de las unidades de } \\
\text { aprendizaje en el proyecto. Argumenta su intervención en el proyecto considerando la matriz } \\
\text { de competencias }\end{array}$ \\
\hline & $\mathrm{COM}$ & $\begin{array}{l}\text { Expone sus argumentos para establecer la articulación con las demás unidades de } \\
\text { aprendizaje. }\end{array}$ \\
\hline & $\mathrm{T}$ & $\begin{array}{l}\text { Analiza y discute la organización de las unidades de aprendizaje para participar en el } \\
\text { proyecto. Reconoce los conceptos que intervienen. }\end{array}$ \\
\hline \multirow[t]{4}{*}{ Etapa 2} & C & $\begin{array}{l}\text { Interpreta el contenido de las diferentes unidades de aprendizaje. } \\
\text { Analiza las diferentes propuestas del grupo. } \\
\text { Sintetiza las diferentes aportaciones. }\end{array}$ \\
\hline & $C L$ & $\begin{array}{l}\text { Describe los diferentes puntos de vista del grupo y de los docentes. } \\
\text { Argumenta la toma decisiones de las distintas aportaciones. }\end{array}$ \\
\hline & $\mathrm{COM}$ & Expone sus afirmaciones y las defiende frente al grupo de trabajo. \\
\hline & $\mathrm{T}$ & Argumenta con claridad el tema del proyecto y la hipótesis ante el grupo. \\
\hline \multirow[t]{4}{*}{ Etapa 3} & C & $\begin{array}{l}\text { Analiza la organiza del cronograma de actividades de apegado a los objetivos establecidos } \\
\text { en las reuniones con la planta docente (plan de curso). } \\
\text { Interpreta la información recopilada. } \\
\text { Abstrae el desarrollo lógico de teorías formales y sus relaciones. }\end{array}$ \\
\hline & $C L$ & $\begin{array}{l}\text { Describe la organización de los contenidos a los docentes. } \\
\text { Argumenta su impacto en el proyecto. }\end{array}$ \\
\hline & COM & Expone sus conclusiones. \\
\hline & $\mathrm{T}$ & Considera las ideas previas, elabora diseños experimentales y observacionales. \\
\hline \multirow[t]{4}{*}{ Etapa 4} & C & $\begin{array}{l}\text { Discute y argumenta con el grupo de docentes para establecer los contenidos que apoyarán } \\
\text { el desarrollo del proyecto. }\end{array}$ \\
\hline & $\mathrm{CL}$ & $\begin{array}{l}\text { Selecciona, utiliza y evalúa las tecnologías de la comunicación e información como recurso } \\
\text { de enseñanza aprendizaje }\end{array}$ \\
\hline & $\mathrm{COM}$ & $\begin{array}{l}\text { Establece diferentes acercamientos para las relaciones con las otras unidades de } \\
\text { aprendizaje, de acuerdo con el programa de su unidad de aprendizaje. }\end{array}$ \\
\hline & $\mathrm{T}$ & $\begin{array}{l}\text { Establece el cronograma de actividades de acuerdo a la planeación por unidad de } \\
\text { aprendizaje, considerando el material didáctico pertinente al contexto. }\end{array}$ \\
\hline \multirow[t]{4}{*}{ Etapa 5} & C & $\begin{array}{l}\text { Interpreta y analiza los ambientes favorables y desafiantes para el aprendizaje. } \\
\text { Analiza y discute el avance del proyecto }\end{array}$ \\
\hline & $\mathrm{CL}$ & Describe las actividades frente al grupo. \\
\hline & COM & $\begin{array}{l}\text { Realiza discusiones plenarias con el equipo de docentes para analizar los avances. } \\
\text { Explica las actividades entre las unidades de aprendizaje, calendarizar las sesiones para el } \\
\text { control y evaluación participativa. }\end{array}$ \\
\hline & $\mathrm{T}$ & Fomenta el trabajo en equipos y la discusión del proyecto. \\
\hline \multirow[t]{4}{*}{ Etapa 6} & C & $\begin{array}{l}\text { Sintetiza el trabajo desarrollado en el proyecto. } \\
\text { Analiza e interpreta los resultados a través de la exposición de los estudiantes }\end{array}$ \\
\hline & $\mathrm{CL}$ & $\begin{array}{l}\text { Describe y argumenta los obstáculos y fortalezas del proyecto desde su unidad de } \\
\text { aprendizaje. }\end{array}$ \\
\hline & COM & $\begin{array}{l}\text { Explica el método. } \\
\text { Explica los procesos y resultados }\end{array}$ \\
\hline & T & $\begin{array}{l}\text { Presenta argumentos y conclusiones de los hallazgos identificados en el proyecto ante los } \\
\text { docentes. }\end{array}$ \\
\hline
\end{tabular}




\section{CONCLUSIONES}

El proceso de aprendizaje del docente durante la experiencia presentó avances y retrocesos, principalmente durante la interpretación de las situaciones obtenidas y de las estrategias didácticas que se llevarían a cabo en el aula. El trabajo de equipo contribuyó para que los profesores fortalecieran la comunicación entre las diferentes asignaturas para organizar contenidos y establecer estrategias ha seguir durante el desarrollo del proyecto, así mismo las discusiones en plenarias favorecen el debate y la defensa de argumentos en un ambiente de análisis y razonamiento y la forma de organizar las actividades en el seminario, favorecieron la exposición de ideas y conjeturas por parte de los docentes para la re-organización de los contenidos.

El espacio curricular del área tecnológica resultó un ámbito propicio para trabajar este tema, ya que permitió al docente integrar contenidos conceptuales de otros espacios curriculares y relacionar la el área tecnológica con el mundo real.

Durante la experiencia se presentaron situaciones en las cuales el docente claudicó, no obstante es necesario continuar el trabajo interdisciplinario para establecer comunicación permanente con y entre los profesores teniendo un fin común; el desarrollo integral del estudiante.

\section{AGRADECIMIENTOS}

Las autoras agradecen el apoyo otorgado por la Secretaria de Investigación y Posgrado a través de las investigaciones con números de registro; 20110397 y 20111060.

\section{REFERENCIAS}

Acevedo, J. A., Conocimiento Didáctico del Contenido para la Enseñanza de la Naturaleza de la Ciencia (II): Una Perspectiva. (2009). Recuperado 29 de agosto de 2012 de http://redalyc.uaemex.mx/redalyc/pdf/920/92012978001.pdf

Astudillo C. Rivarosa A. y Ortiz F., Formas de pensar la enseñanza en ciencias. Un análisis de secuencias didácticas. (2011)Recuperado el 05 de agosto de 2012 de http://www.saum.uvigo.es/reec/volumenes/volumen10/REEC_10_3_10.pdf

Aravena M., Caamaño C. y Giménez J., Modelos Matemáticos a través de Proyectos, Revista Latinoamericana de Investigación Educativa, 11(1), 49-92 (2008).

Camarena, P., La Matemática en el contexto de las ciencias, Revista Innovación Educativa, IPN. 9(46), (2009).

Echeverría, J., Ciencia y Valores, Barcelona, Ediciones destino, S.A. 2002.

Enríquez, M. Enríquez, S. y Pizano S. Proyecto de Aula, una Propuesta Metodológica. (2007). Recuperado el 23 de agosto de 2012 de http://www.ciie.cfie.ipn.mx/2domemorias/documents/m/m14a/m14a_33.pdf

Instituto Politécnico Nacional. Materiales para Reforma. Un Nuevo Modelo educativo para el IPN. México: IPN. (2004).

Korthagen, F.A.J. The gap between research and practice revisited. Educational Research and Evaluation, 13(3), 303-310 (2007).

Lacueva A., Formando docentes integrales que quieran y puedan enseñar ciencia y tecnología. (2010).

Recuperado el 20 de agosto de 2012 de

http://www.saum.uvigo.es/reec/volumenes/volumen9/ART2_Vol9_N2.pdf

Pro Bueno A. y Ezquerra A.,¿Qué ropa me pongo?. Cómo percibe le alumnado los contenidos científicos con audiovisuales. (2008). Recuperado el 02 de septiembre de 2012 de http://www.investigacionenlaescuela.es/articulos/64/R64_6.pdf 
